\title{
PERFORMANCE EFFECTS OF SETUP TIME REDUCTION WITH AND WITHOUT DECISION VARIABLE RE-OPTIMIZATION: A SIMULATION-OPTIMIZATION STUDY
}

\author{
Chandandeep S. Grewal \\ Silvanus T. Enns \\ Paul Rogers \\ Department of Mechanical and Manufacturing Engineering \\ University of Calgary \\ Calgary, AB, T2N 1N4, CANADA
}

\begin{abstract}
This study investigates the benefits of setup time reduction in a simple, capacity-constrained supply chain using reorder point replenishment. A discrete-event simulation model was developed and linked to an optimization engine for simulationoptimization experiments. Performance trade-off curves between total inventory and customer service levels were generated under different setup times. Comparisons were made with and without re-optimizing the reorder point and lot size decision variables after reducing setups. It was found that the benefits of setup reduction can be amplified with re-optimizing. Analysis of variance (ANOVA) was used to analyze the main and interaction effects. The behavior of the optimal decision variables under setup time reduction was also examined. Insights should provide guidance for industrial practitioners wishing to maximize the benefits of setup time reduction.
\end{abstract}

\section{BACKGROUND}

Setup time reduction has become well accepted as a way to improve manufacturing efficiency in batch production environments where multiple product types are produced on common machines and each lot of product incurs a new setup. The fact that setup time reduction frees machine time and therefore decreases lot flowtime or increases potential throughput is wellunderstood. However the interaction between setup time reduction and optimal lot sizing is more difficult to evaluate. As well, interactions between setup time reduction and optimal replenishment decision variables, such as lot size and reorder point settings, have not been extensively studied.

A number of researchers have conducted studies related to setup time reduction. Spence and Porteus (1987) studied setup time reduction using deterministic demand assumptions and an extended economic-order-quantity (EOQ) model. This research clearly showed setup time reduction not only freed up machine time but also allowed smaller lot sizes to be used. The net effect was to further reduce lot flowtimes and system inventory.

Another approach has been to consider stochastic demand for lots of products using queuing relationships. Usually a Poisson arrival process, with negative exponential lot interarrival times, is assumed to ensure assumptions of independent arrivals are not violated. Yang and Deane (1993) used an M/G/1 queuing model and concluded that both optimal lot sizes and flowtime variance decreased with reduced setup times.

Queuing relationships can also be applied to analyze cyclical production problems with multiple product types if Poisson arrivals are assumed. Sarkar and Zangwill (1991) and Samaddar and Hill (2007) examined setup time reduction for specific product types and how this affected overall setup time variability. It was found that setup time reduction can actually cause deterioration in performance in some circumstances if it induces increased variability. This research did not seek to optimize lot sizes.

In complex environments with multiple processing stages interarrival times are not likely to be independent and queuing theory has limited application. In this case simulation can be used to study the effects of setup time reduction. Li (2003) examined the effects reducing both the mean and variance of setup times. It was concluded that interactions with the configuration of the shop, which dictates flow patterns, are also important. 
In cases where interarrivals are not independent finding optimal lot sizes is challenging. Enns (2007) used simulation along with response surface techniques to determine optimal lot sizes. Non-linear optimization was applied to find optimal lot sizes in both a single-stage reorder point and Kanban system.

This research seeks to extend the field by examining setup time reduction with and without the re-optimization of decision variables, such as reorder points and lot sizes. The methodology used is also different from that used in previous studies. The use of simulation-optimization means that assumptions regarding independent arrivals are not required and a more realistic production scenario can be dealt with. It is also a much more efficient way of finding optimal settings as compared to using response surface methods. In this research a continuous-review reorder point replenishment system is dealt with, where both the lot sizes and reorder points can be optimized. A primary objective is to study the effect of setup time reduction with and without re-optimization of the decision variables, and to determine the importance of changing lot sizes and reorder points along with setup times. A second objective is to study the behavior of the optimal decision variable settings as setup times are reduced.

The remainder of the paper is organized as follows. Section 2 presents the research scenario, including assumptions. Section 3 describes the research methodology and experimental designs. Following this the results and analysis are given in Section 4. Finally, the conclusions of this research are presented.

\section{2}

\section{RESEARCH SCENARIO}

A simple, capacity-constrained supply chain was considered for evaluating a continuous-review reorder point replenishment system. Batch production was assumed to take place at a manufacturer which supplied a warehouse from which customer demand was filled.

\subsection{Experimental Supply Chain}

This supply chain, shown in Figure 1, consisted of customers, a warehouse for finished goods inventory and a manufacturing plant. There were two types of product produced. These products were not interchangeable with respect to customer demand but did have the same processing requirement characteristics. Raw materials for the manufacturing process were assumed to be available from the supplier at all times.

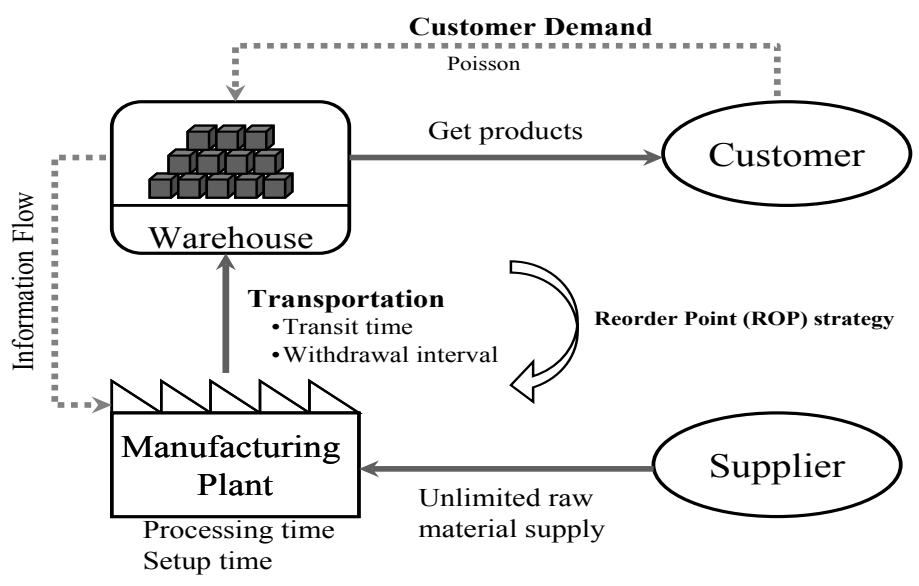

Figure 1: Supply chain scenario

Customers demanding each type of product arrived according to a Poisson process. Each customer demanded only one unit. If there was inventory of the required product type in stock the customer demand was filled. Otherwise the demand was backordered and filled once inventory became available. There were assumed to be no lost sales.

The replenishment decision variables were the reorder point and reorder quantity, or lot size, for each product type. This lot size was also the production batch size. The inventory position, which was continuously reviewed, was calculated on the basis of current finished goods inventory, unfilled orders released to the manufacturer and customer backorders. Once the inventory position fell to the reorder point, an order was initiated. The order transmission time was assumed to be 1.2 time units but there was an additional delay for order processing, either at the warehouse or manufacturer. This delay, in time units, was randomly generated from a uniform distribution with parameters $(0,4)$. 


\section{Grewal, Enns and Rogers}

Once the orders were received at the manufacturer they were processed in first-come-first-served (FCFS) priority. The manufacturer was considered to have only one processing stage. Each unit in the order had a processing time of 0.015 time units. These values were the same for both product types and were deterministic. A setup was required between each order (or batch), regardless of the sequence of product types being produced. This setup time was considered to be an experimental factor.

Once any order was completed at the manufacturer it had to wait for a transporter to ship it to the warehouse. Transporters were released from the manufacturer at fixed intervals of 4 time units and could carry zero, one or multiple lot-size orders of any product type. The downstream travel time distribution was triangular with parameters $(0.6,1.2,1.8)$.

A discrete-event simulation model of this scenario was developed using Arena 12.0® (Kelton, Sadowski and Sturrock 2007). This model was designed to be highly parametric, flexible and easy to understand. The model was also linked to OptQuest ${ }^{\circledR}$ for optimization purposes.

\section{RESEARCH METHODOLOGY}

The initial objective was to determine the optimal reorder point and lot size decision variable settings at different lot setup time and delivery service levels. The performance measures and simulation-optimization procedure are described in the next two sub-sections. Following this the experimental designs used to study the effects of setup time reduction with and without decision variable re-optimization are presented.

\subsection{Performance Measures}

The main performance criteria were related to inventory levels and customer delivery performance, along with the tradeoffs between them. The two measures used to address these criteria were total inventory $(T I)$ and the customer fill rate (SL).

The total inventory measure, given as Equation (1), included finished goods and work-in-process inventory. Work-inprocess inventory was assumed to include all lots waiting to be processed, at the processing station, waiting for a transporter or in transit to the warehouse.

$$
T I=\sum_{i=1}^{n} W I P_{i}+\sum_{i=1}^{n} F G_{i}
$$

where:

$$
\begin{array}{ll}
T I & =\text { total inventory of the system } \\
W I P_{i} & =\text { work-in-process inventory for product type } i \\
F G_{i} & =\text { finished goods inventory for product type } i \\
n & =\text { total Number of product types ( } n=2 \text { in this case) }
\end{array}
$$

The service level measure, given as Equation (2), was defined to be the proportion of customer demand filled from stock for product type $i$. This is sometimes referred to as the fill rate.

$$
S L_{i}=\left(\frac{\text { Product type } i \text { demand filled directly from stock }}{\text { Total product type } i \text { demand }}\right)
$$

These two measures were used as the basis for optimizing the reorder point $\left(O P_{i}\right)$ and lot size $\left(L S_{i}\right)$ decision variables for each product type $i$.

\subsection{Simulation-optimization}

Experiments in this research were carried out using simulation-based optimization. Equation (3) shows the optimization formulation along with the objective function, decision variables and constraints.

$$
\operatorname{Min} T I\left(O P_{i}, L S_{i}\right)=\sum_{i=1}^{n} W I P i+\sum_{i=1}^{n} F G i
$$

Subject to:

$$
\begin{aligned}
& S L_{i}\left(O P_{i}, L S_{i}\right) \geq S L_{p} \quad \text { for } i=1,2 \ldots \ldots n \\
& l\left(O P_{i}\right) \leq O P_{i} \leq u\left(O P_{i}\right) \\
& l\left(L S_{i}\right) \leq L S_{i} \leq u\left(L S_{i}\right) \\
& O P_{i}, L S_{i} \text { are integers }
\end{aligned}
$$

where:

TI = total inventory of the system 


\section{Grewal, Enns and Rogers}

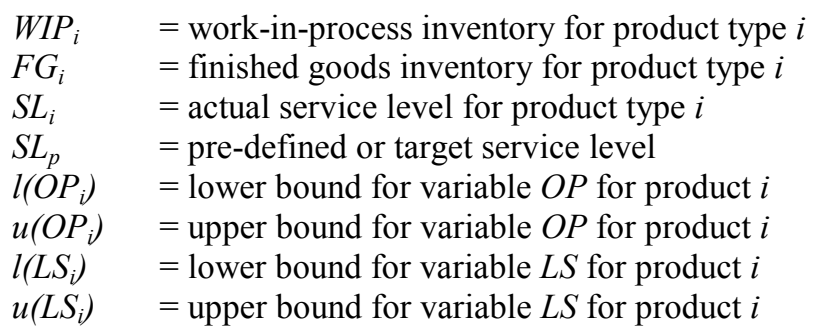

The objective function was to minimize the required inventory subject to achieving some target customer service level $\left(S L_{p}\right)$. This customer service level was changed from run to run in order to generate results for a performance trade-off curve. The values of $l\left(O P_{i}\right), u\left(O P_{i}\right), l\left(L S_{i}\right)$ and $u\left(L S_{i}\right)$ are simply bounds that the user must specify in order to restrict the search space over which OptQuest ${ }^{\circledR}$ looks for the optimal decision variables. The observed service level, $S L_{i}$, was constrained to be greater than or equal to the target, $S L_{P}$, since it is impossible to achieve the exact service level desired when the decision variables are constrained to be integers.

\subsection{Experimental Design}

Experimentation consisted of two stages. The first stage was to find the optimal decision variable settings for various factor level combinations. This was done using simulation-optimization. The second stage consisted of running simulation experiments at the same factor level combinations with and without using optimal decision variables. The experimental design for this stage included replication to facilitate statistical analysis.

The experimental design for the first stage consisted of two factors. These were the lot setup time $(S T)$ and the target service level $(S L)$. The factor levels are shown in Table 1. The total number of combinations in this experimental design was 24 .

Table 1: Factor settings for $O P$ and $L S$ optimization experiments

\begin{tabular}{|c|c|c|}
\hline Factor & Type & Levels \\
\hline$S T$ & Numeric, fixed & $0.25,0.35 ., 0.45,0.55,0.65,0.75$ \\
\hline$S L$ & Numeric, fixed & $0.80,0.85,0.90,0.95$ \\
\hline
\end{tabular}

Simulation-optimization experiments were run for each of the combinations. Arena ${ }^{\circledR}$ and OptQuest ${ }^{\circledR}$ were used together to find the optimal reorder point $\left(O P_{i}\right)$ and lot size $\left(L S_{i}\right)$ values at each combination. Optimality was based on minimizing the total inventory $(T I)$ subject to the service level constraint $\left(S L_{p}\right)$, as given in Equation (3). In other words, while the setup time (ST) was set at different levels in the simulation model, the service level, $S L_{P}$, was treated as a constraint during optimization.

The warmup period for each simulation run was 1500 time units. It was confirmed that this was sufficient for the system to reach steady-state conditions. Data was collected over 50,000 time units for every run. These simulation-optimization experiments yielded optimal reorder points $\left(O P_{i}\right)$ and lot sizes $\left(L S_{i}\right)$ for each product type $i$ at each of the 24 setup time and service level combinations.

The experimental design for the second stage consisted of three factors. The first two factors were the same as for the first stage. The third factor was whether or not optimal decision variables were being used along with reduced setup times. This factor was called ReOpt and was run at two levels. The first level (-1) indicated that optimal $O P_{i}$ and $L S_{i}$ decision variables were used. The second level $(+1)$ indicated optimal $O P_{i}$ and $L S_{i}$ values for a base case with a setup time of 0.75 were being used. In other words, experiments at the +1 level were rerun at each combination of $S T$ and $S L$ factor levels using the optimal $O P_{i}$ and $L S_{i}$ values at the given service level related to a setup time of 0.75 .

The second stage experiments involved using the reorder points $\left(O P_{i}\right)$ and lot sizes $\left(L S_{i}\right)$ predetermined in the first set of experiments. Therefore optimization was not required and Arena ${ }^{\circledR}$ could be run without OptQuest ${ }^{\circledR}$. These simulation runs were relatively fast so the data collection period was increased to 100,000 time units. As well, all 48 combinations of experimental settings ( $6 S T * 4 S L * 2$ ReOpt levels) were run for 5 replications to facilitate later statistical analysis. Common random numbers were used as a variance reduction technique.

\section{$4 \quad$ RESULTS AND ANALYSIS}

This section first looks at the performance effects of setup time reduction with and without decision variable re-optimization. Evaluation is presented using trade-off curves between inventory and delivery service levels. Next, the main and interaction 
effects are examined statistically. Finally, the effects of setup time reduction on the optimal decision variable settings is presented.

\subsection{Setup time reduction effects on performance}

Figure 2 illustrates the performance trade-off curves between total inventory $(T I)$ and the service level $(S L)$, plotted separately for the six different setup times $(S T)$. The upper left plot shows the base case after optimization. The upper curve on each additional plot is the performance with re-optimization of the decision variables $\left(O P_{i}\right)$ and $\left(L S_{i}\right)$ and the lower curve is without re-optimization. Each point on each curve is the average for the five replications. Points along the curves moving up and toward the right represent increased service level $(S L)$ targets.

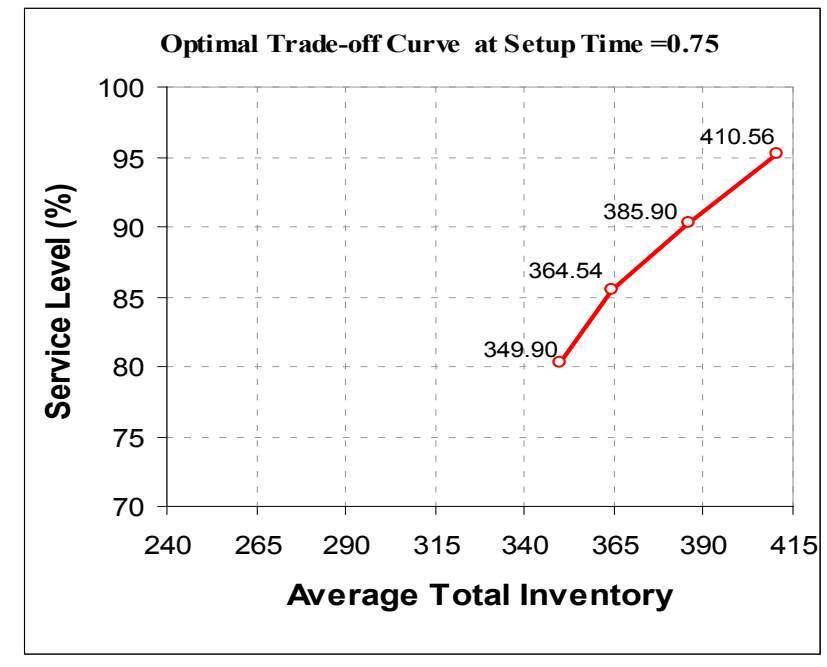

(a)

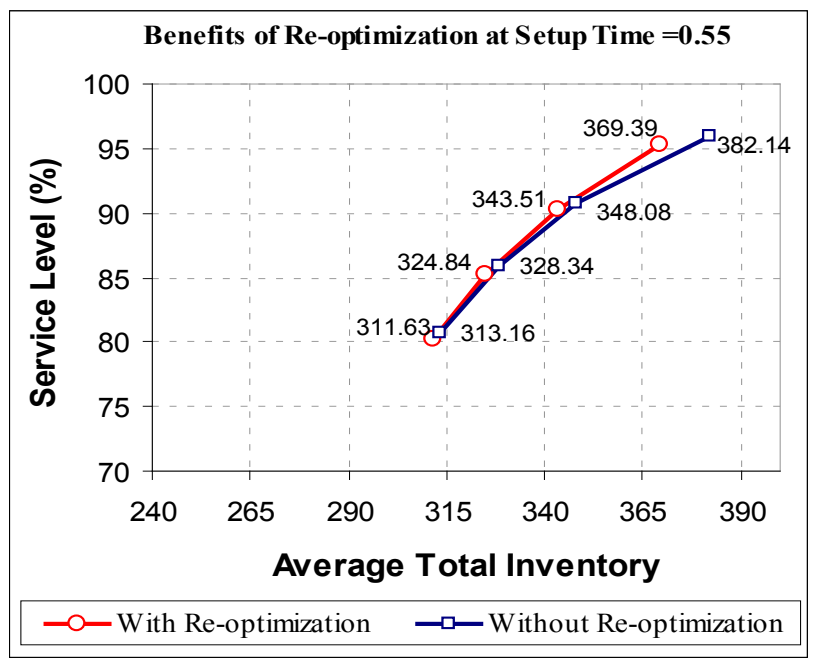

(c)

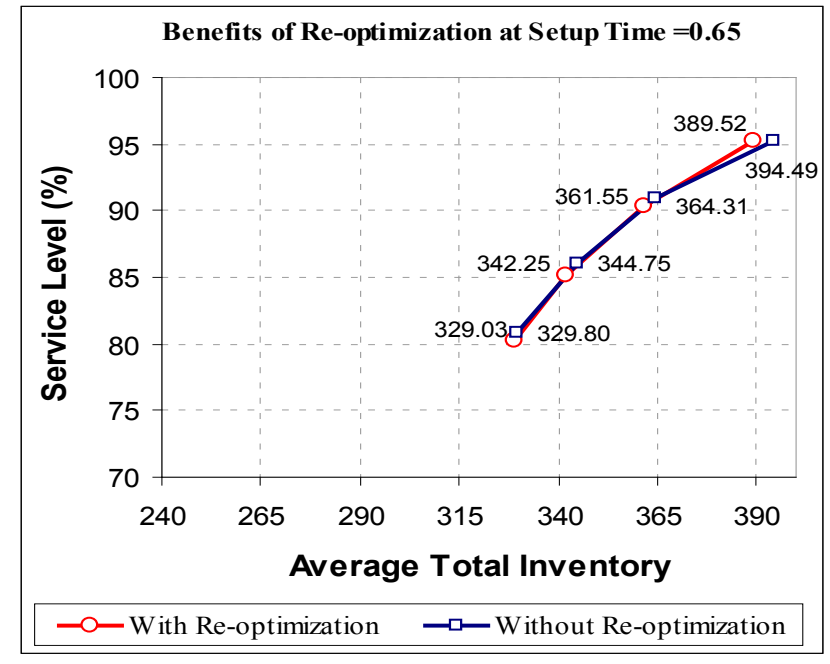

(b)

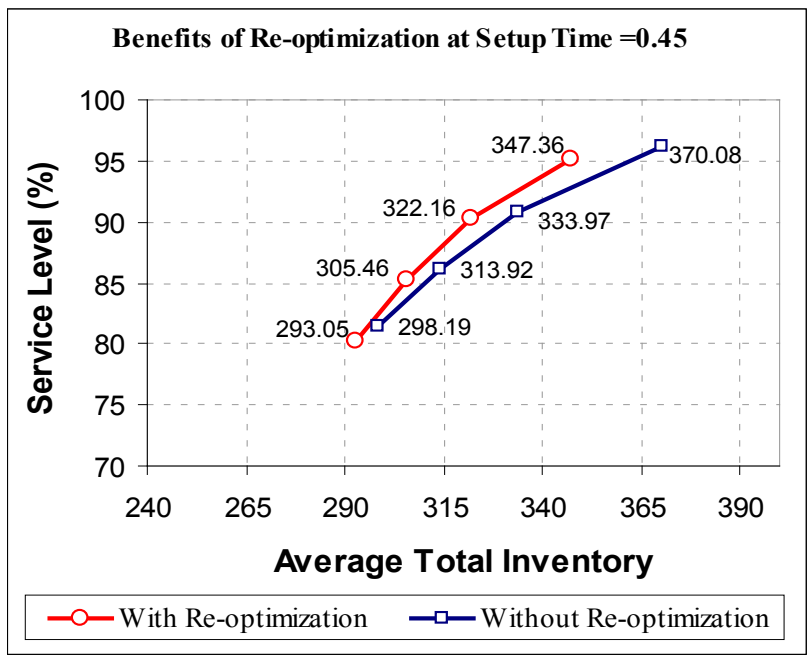

(d) 


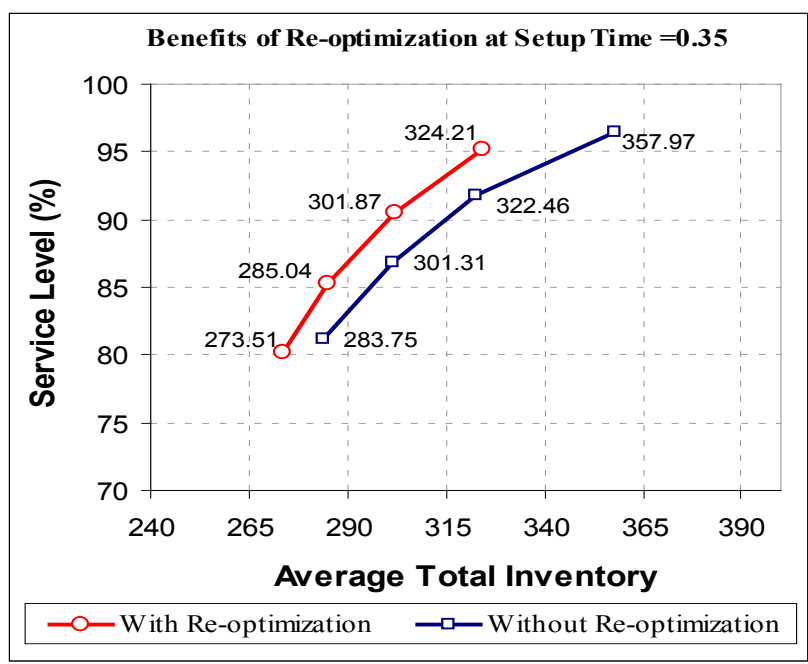

(e)

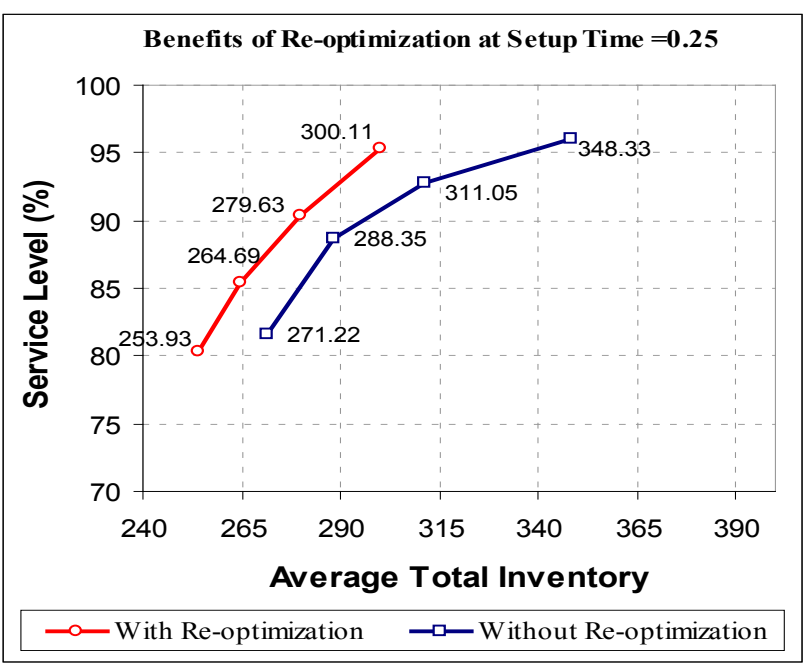

(f)

Figure 2: Performance trade-off curves with and without re-optimization

The plots in Figures 2(b) through 2(f) clearly show that setup time reduction improves performance. This is true even if the decision variables are not re-optimized. Comparing the curves with the square markers across the different plots shows the curves shift towards the left as the setup times decrease. However, the biggest benefit is realized if the decision variables are also re-optimized as setup times are reduced. The curves with re-optimization, indicated by the circular markers, clearly dominate the performance of the curves without re-optimization. This is especially true at the very low setup time values.

The other interesting observation is that the differences in performance between re-optimizing and not re-optimizing the decision variables increases with increasing service level targets. In other words, the curves are further apart towards the top of the plot. This indicates it is especially beneficial to re-optimize reorder points and lot sizes as setup times are reduced if high delivery performance levels are desired.

Additional analysis was also performed using only the results with optimal decision variable settings. Figure 3 shows the effects of setup time $(S T)$ reduction on work-in process $(W I P)$ inventory and finished goods inventory (FGI). For clarity, results are shown for only the $80 \%$ and $95 \%$ service levels $(S L)$. As $S T$ is reduced, both $W I P$ and $F G I$ are also reduced. However, the magnitude of WIP reduction is greater than the magnitude of $F G I$ reduction. It is also observed that at each setup time the WIP is approximately the same for both service levels. Figure 4 presents the effects of setup time reduction on backorder waiting times. The time that customers spend waiting for backordered items decreases as the setup time is reduced.

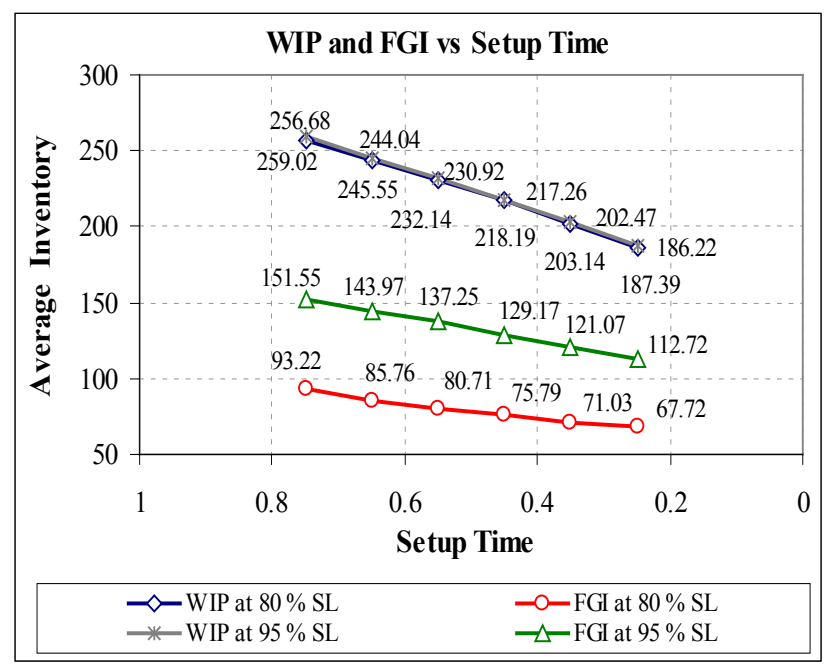

Figure 3: Effect of $S T$ on $W I P$ and $F G I$

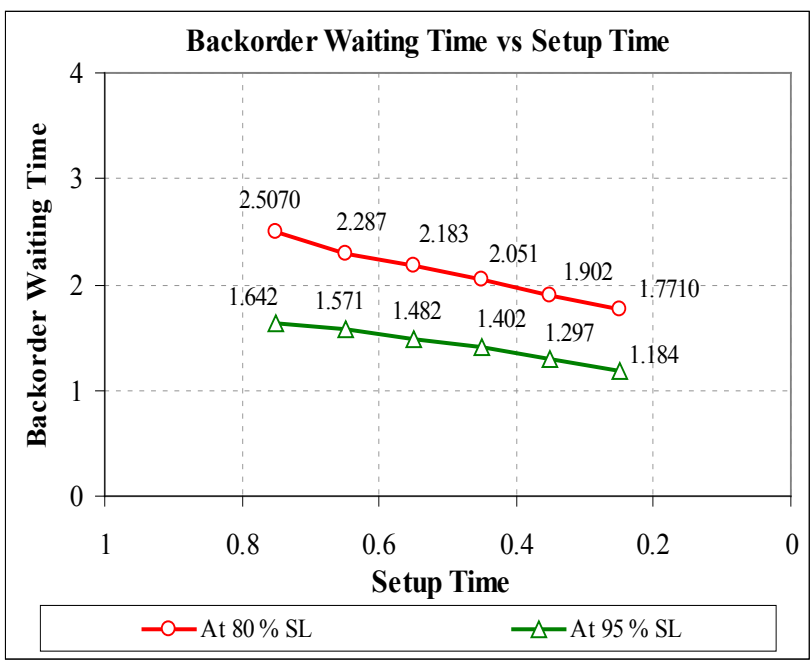

Figure 4: Effect of $S T$ on backorder waiting time 


\subsection{Setup time main and interaction effects}

The graphical results indicate that there are interaction effects between the various factor settings. A $2^{3}$ full factorial design was used to gain statistical confirmation using analysis-of-variance (ANOVA). Only two levels of $S T$ and $S L$ were chosen to simplify interpretation. Table 2 shows both the factor levels and the total inventory (TI) response. These results are based on 5 replications. The total number of observations used in this analysis was $40(2 * 2 * 2 * 5)$.

Table 2: Full-factorial design with factors, levels and performance measures

\begin{tabular}{|c|c|c|c|}
\hline \multirow{3}{*}{$\begin{array}{c}\text { Factor 1: } \\
\text { Setup Time }(S T)\end{array}$} & \multirow{3}{*}{$\begin{array}{c}\text { Factor 2: } \\
\text { Service Level }(S L)\end{array}$} & \multicolumn{2}{|c|}{ Average Total Inventory $(T I)$} \\
\hline & & \multicolumn{2}{|c|}{ Factor 3: Re-optimization ( $\mathrm{ReOpt})$} \\
\hline & & $\begin{array}{l}\text { With Re-optimization } \\
\quad(\text { ReOpt }=-1)\end{array}$ & $\begin{array}{l}\text { Without re-optimization } \\
(\operatorname{ReOpt}=+1)\end{array}$ \\
\hline \multirow{2}{*}{$0.35(-1)$} & $85 \%(-1)$ & 285.037 & 301.312 \\
\hline & $95 \%(+1)$ & 324.214 & 357.968 \\
\hline \multirow[t]{2}{*}{$0.65(+1)$} & $85 \%(-1)$ & 342.247 & 344.747 \\
\hline & $95 \%(+1)$ & 389.519 & 394.486 \\
\hline
\end{tabular}

The ANOVA results, obtained using Minitab ${ }^{\circ}$, are shown in Table 3. These results show that all main, two-way interaction and three-way interaction effects were significant at the 95\% confidence level. In other words, the $p$-values are less than 0.05 in all cases. The residual analysis is shown graphically in Figure 5. The ANOVA assumptions of normally distributed errors and equal within-group variances appear not to have been violated. Therefore this ANOVA model was judged satisfactory.

Table 3: Analysis of variance (ANOVA) for $T I$

\begin{tabular}{lcccccc}
\hline Source & DF & Seq SS & Adj SS & Adj MS & F & P \\
\hline$S T$ & 1 & 26650.6 & 26650.6 & 26650.6 & 582086 & 0.000 \\
$S L$ & 1 & 22281.6 & 22281.6 & 22281.6 & 486661.5 & 0.000 \\
$\operatorname{ReOpt}$ & 1 & 1786.6 & 1786.6 & 1786.6 & 39021.4 & 0.000 \\
$S T^{*} S L$ & 1 & 5.1 & 5.1 & 5.1 & 111.1 & 0.000 \\
$S T^{*} \operatorname{ReOpt}$ & 1 & 1356.8 & 1356.8 & 1356.8 & 29634.02 & 0.000 \\
$S L^{*} \mathrm{ReOpt}$ & 1 & 359.3 & 359.3 & 359.3 & 7846.92 & 0.000 \\
$S T^{*} \mathrm{SL} * \mathrm{ReOpt}$ & 1 & 75.4 & 75.4 & 75.4 & 1646.35 & 0.000 \\
Error & 32 & 1.5 & 1.5 & 0 & & \\
Total & 39 & 52516.7 & & & & \\
\hline
\end{tabular}

Figures 6 and 7 show plots of the main and interaction effects. It is obvious from these plots and from the ANOVA table that setup times $(S T)$ and service levels $(S L)$ have the greatest impact on the total inventory $(T I)$ required. The most important two-way interaction is between $S T$ and ReOpt. The two-way interaction plot confirms that re-optimization is increasingly important as setups are further reduced from the base case. The two-way interaction between $S L$ and ReOpt is also shown to be important. The plot confirms that re-optimization is especially important to minimize inventory requirements if high service levels are being targeted. The two-way interaction between the $S T$ and $S L$ would not appear to be very important, judging by the parallel lines, even though the ANOVA results showed it to be statistically significant. 


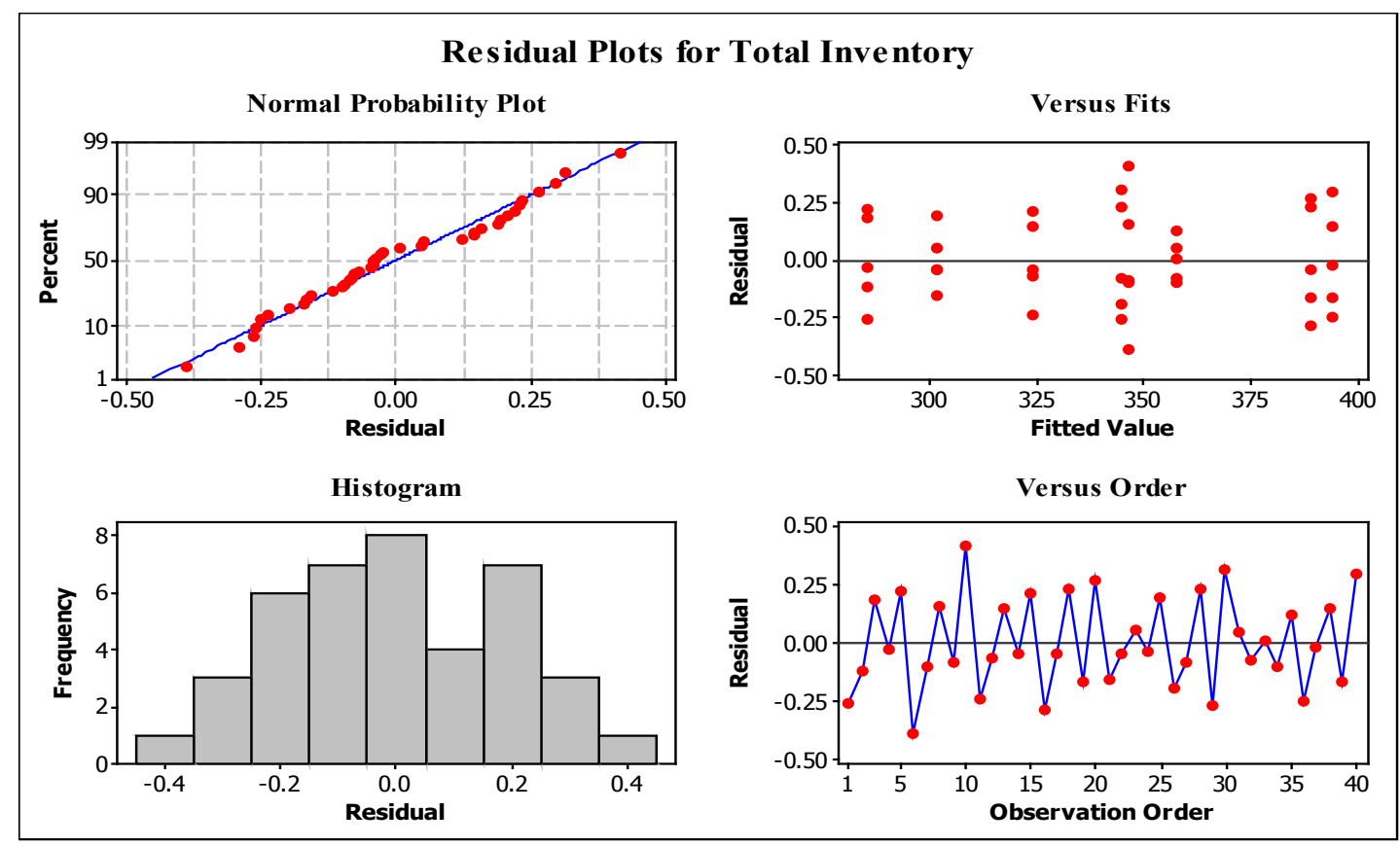

Figure 5: Residual plots for $T I$ as a response

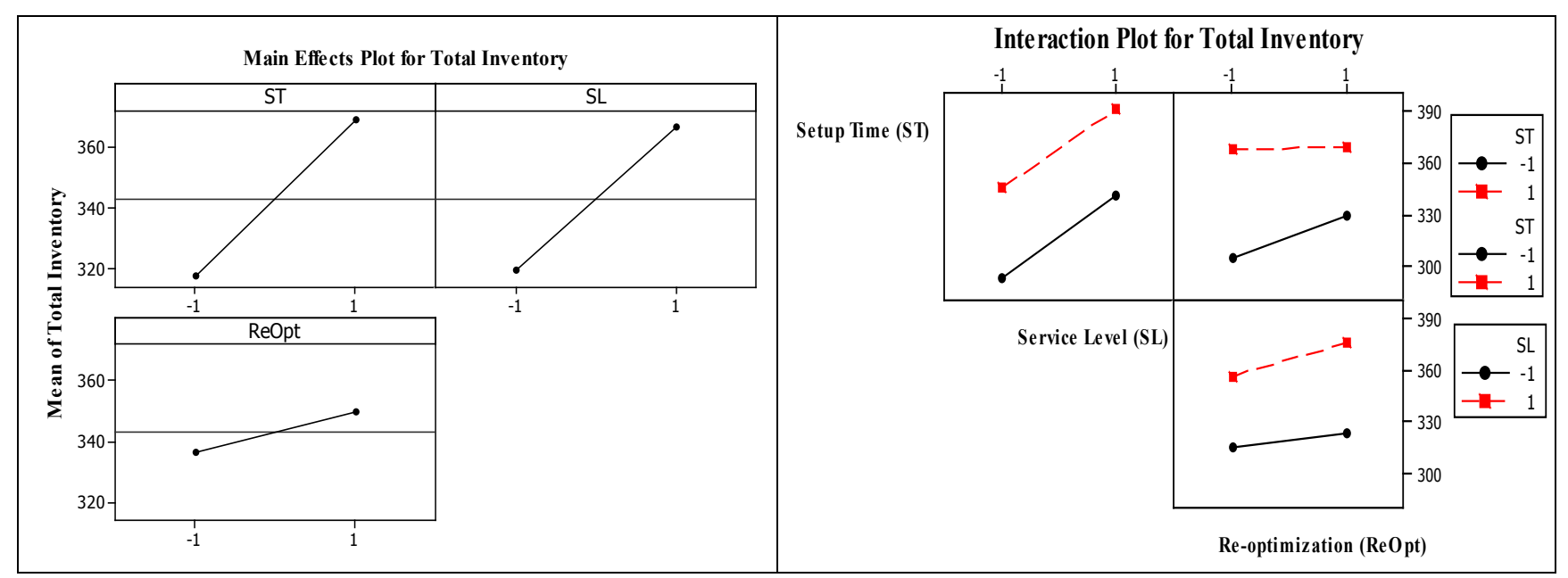

Figure 6: Main effects of factors $T I$

Figure 7: Interaction effects of factor levels on $T I$

\subsection{Setup time reduction effects on optimal decision variables}

Finally, the optimal lot sizes $\left(L S_{i}\right)$ and reorder points $\left(O P_{i}\right)$ were examined as a function of setup time reduction. Figures 8 and 9 show the results, at the $80 \%$ and $95 \%$ service levels, for the lot sizes and reorder points respectively.

It can readily be observed from Figure 8 that the optimal lot sizes decrease considerably as setups are reduced. However, in comparing Figures 8(a) and 8(b) it can be seen that the service level target has less impact on the optimal lot sizes. The behavior is quite different for the optimal reorder points, shown in Figure 9. In this case the reorder points decrease only slightly with decreasing setup times. However, in comparing Figures 9(a) and 9(b) it can be seen that the service level target has a significant impact on the optimal reorder points. As the targeted service level target goes up, much higher reorder points are required. 


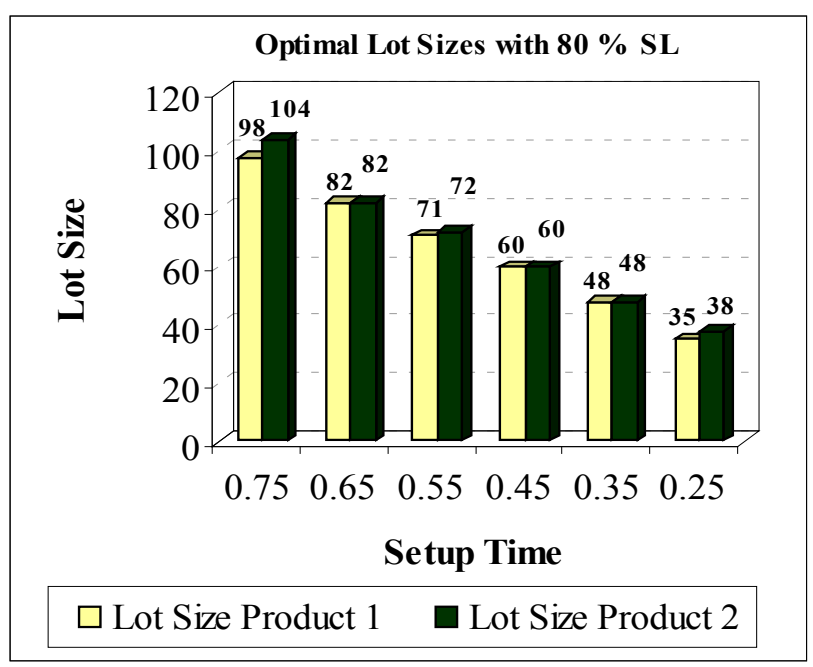

(a)

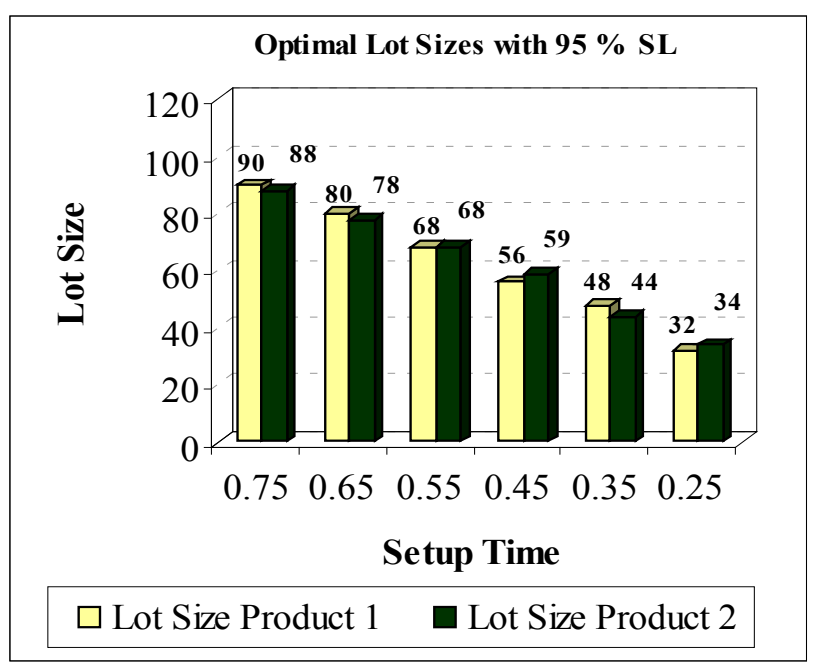

(b)

Figure 8: Behavior of optimal lot sizes with setup time reduction at (a) $80 \% S L$ (b) $95 \% S L$

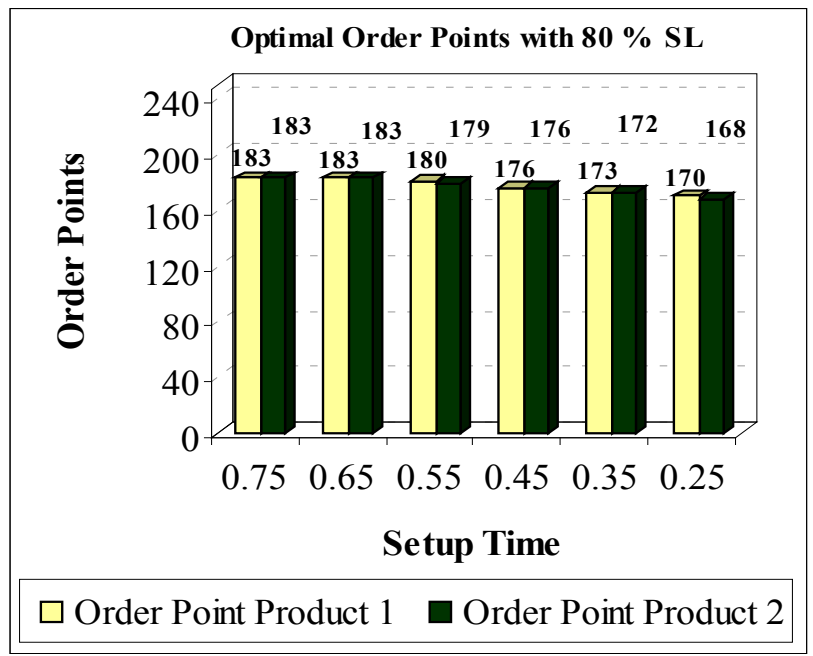

(a)

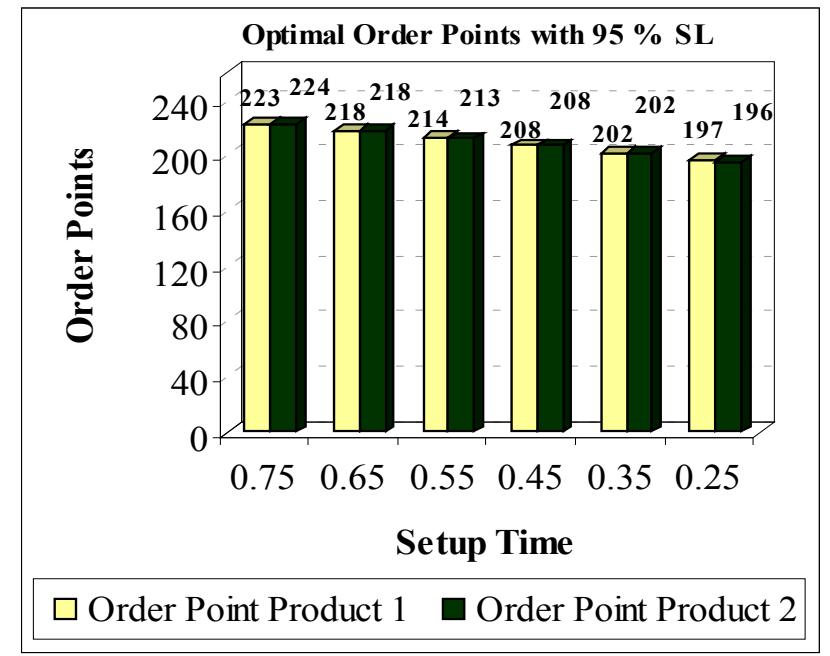

(b)

Figure 9: Behavior of optimal reorder points with setup time reduction at (a) $80 \% S L$ (b) $95 \% S L$

\section{CONCLUSIONS}

This study has evaluated the performance of a simple, capacity-constrained supply chain under setup time reduction. Performance has been evaluated with and without re-optimization of the lot size and reorder point decision variables. Simulationoptimization was used to find optimal decision variable settings across a range of desired delivery performance targets. It was found that setup time reduction alone reduces the total system inventory required to meet a specific customer fill rate. However, this inventory reduction can be amplified if the decision variables are re-optimized as well. ANOVA results confirmed the statistical significance of the benefits of setup time reduction as well as the presence of interaction effects. The results also revealed that the optimal lot sizes decrease significantly with reduced setups. In addition, lot sizes were found to be slightly smaller at increased service level targets. In contrast, the optimal reorder points are mainly a function of the desired delivery service level and setup reduction has less impact.

The methodology used in this study could be extended to more complex scenarios. It could be used to study the effects of setup time variability on performance as well. The methodology may also be of interest to determine if the benefits of setup time reduction change under different replenishment assumptions. For example, setup time reduction under a continuousreview reorder point system could be compared with setup time reduction under a single-card Kanban system. 


\section{ACKNOWLEDGMENTS}

Drs. Enns and Rogers wish to thank NSERC for Discovery Grant funding to support the research reported. Additionally, Mr. Grewal wishes to thank the Killam Trusts for scholarship funding.

\section{REFERENCES}

Enns, S. T. 2007. Pull replenishment performance as a function of demand rates and setup times under optimal settings. In Proceedings of the 2007 Winter Simulation Conference, ed. S. G. Henderson, B. Biller, M.-H. Hsieh, J. Shortle, J. D. Tew, and R. R. Barton, 1624-1632. Piscataway, New Jersey: Institute of Electrical and Electronics Engineers, Inc.

Kelton, W.D., R.P. Sadowski and D.T. Sturrock. 2007. Simulation with Arena; Fourth edition, New York, McGraw-Hill.

Li, J. W. 2003. Improving the performance of job shop manufacturing with demand-pull production control by reducing setup / processing time variability. International Journal of Production Economics 84: 255-270.

Samaddar, S. and C.A. Hill. 2007. Controlling adverse effects on work in process inventory while reducing machine setup time. European Journal of Operational Research 180: 249-261.

Sarkar, D. and W. I. Zangwill. 1991. Variance effects in cyclic production systems. Management Science 37: $444-453$.

Spence, A. M. and E.L. Porteus. 1987. Setup reduction and increased effective capacity source: Management Science 33: 1291-1301.

Yang, J. and R.H. Deane. 1993. Setup time reduction and competitive advantage in a closed manufacturing cell. European Journal of Operational Research 69: 413-423.

\section{AUTHOR BIOGRAPHIES}

CHANDANDEEP SINGH is a doctoral student in the Department of Mechanical and Manufacturing Engineering at the University of Calgary, Canada. He received his Master of Technology in Industrial Engineering from the Indian Institute of Technology, Delhi (India). His areas of interest are supply chain management, operations research and simulation modelling. $\mathrm{He}$ is a member of the Institute of Industrial Engineers (IIE), the Canadian Operational Research Society (CORS), the Indian Society of Mechanical Engineers (ISME), and the Indian Society for Technical Education (ISTE). His email address is <csgrewalducalgary.ca>.

SILVANUS T. ENNS is an Associate Professor at the University of Calgary. He received his PhD from the University of Minnesota. His research interests lie in the development of algorithms to support enhanced MRP performance as well as various aspects of job shop, batch production and supply chain modeling and analysis. He is a Professional Engineer and a member of IIE, the American Production and Inventory Control Society (APICS), and CORS. His email address is <ennseucalgary.ca>.

PAUL ROGERS is an Associate Professor in the Department of Mechanical and Manufacturing Engineering at the University of Calgary. He holds $\mathrm{PhD}$ and M. Eng degrees from Cambridge University in England. His research interests include production planning and control systems, distributed and dynamic scheduling, and models for the design and analysis of healthcare systems. He is a Professional Engineer registered with the Association of Professional Engineers, Geologists, and Geophysicists of Alberta (APEGGA), a member of IIE, APICS, and the Institute for Operations Research and the Management Sciences (INFORMS), and serves on the Editorial Board of the International Journal of Computer Integrated Manufacturing. His email address is <rogerseucalgary . ca>. 